\title{
Spinless Particles in Exponentially Varying Electric and Magnetic Fields
}

\author{
Kenan Sogut and Ali Havare \\ Department of Physics, Mersin University, 33343 Mersin, Turkey \\ Correspondence should be addressed to Kenan Sogut; kenansogut@gmail.com \\ Received 11 July 2014; Accepted 16 September 2014; Published 15 October 2014 \\ Academic Editor: Ming Liu
}

Copyright ( $\odot 2014$ K. Sogut and A. Havare. This is an open access article distributed under the Creative Commons Attribution License, which permits unrestricted use, distribution, and reproduction in any medium, provided the original work is properly cited. The publication of this article was funded by SCOAP S $^{3}$

\begin{abstract}
We investigate the motion of the charged spin- 0 particles subjected to the space-dependent electric and magnetic fields. By selecting the external fields oriented parallel and orthogonal to each other, exact solutions of the motion are obtained for the nonrelativistic and the relativistic cases. The quantized energy spectrum is determined by using the solutions obtained for the motion of the particles and dependence of the energy on the strengths of the electric and magnetic fields is discussed. We compared the energy spectrum of the nonrelativistic and the relativistic particles by using the numerical results obtained for the first few quantum levels.
\end{abstract}

\section{Introduction}

Electric and magnetic fields have lots of fundamental applications used in technology, especially in electrical engineering, electromechanics, particle physics, medical physics, and so forth. Fundamental processes occurring in these applications, developments on the intense particle beams generated by sources such as lasers, nuclear reactors, or accelerators, and appearance of the different configurations of parallel electric and magnetic fields in astrophysics dating from the discovery of the pulsars required noticeable interest on the exact solutions of the relativistic particle equations in external electromagnetic fields. Such efforts have been performed for different configurations of the external fields [1-10]. In these studies, the exact solutions of the nonrelativistic and relativistic wave equations have provided important information regarding the quantum mechanical system. They have been very useful in the interpretation of the physical processes like Compton scattering by a laser source and the Brownian motion, coherent states, and energy levels of electrons. As far as we know there are very few studies on the solution of the wave equation of the spinless particles for the coexistence of the electric and magnetic fields. In one of these, [4] has studied spinless particles for constant electric and magnetic fields.

The purpose of the present paper is to move this subject further. To this end we obtain the exact solutions of the spinless particles for two orientations of exponentially varying electric and magnetic fields that are given by Case (i): $A_{0}=-E_{0} e^{-\alpha z}, A_{1}=\left(B_{0} / \alpha\right) e^{-\alpha y}$ and Case (ii): $A_{0}=E_{0} e^{-\alpha y}$, $A_{1}=\left(B_{0} / \alpha\right) e^{-\alpha y}$, where $E_{0}, B_{0}$ are constants and $\alpha$ is a constant parameter that weakens the strength of the external electromagnetic fields. The first and second cases belong to the parallel and orthogonal fields, respectively. We note that $y$ and $z$ variables are defined in the region $[0, \infty)$ to keep the finite external fields. Such kind of exponentially varying electromagnetic field is encountered in magnetoplasma space [11].

In the following sections, the exact solutions for nonrelativistic and relativistic cases will be obtained, respectively. By comparing the solutions of the nonrelativistic and relativistic wave equations of the spinless particles, contributions coming from the relativistic effects wil be considered and by using the mathematical properties of the wave functions we will obtain the energy spectrum for both cases. 


\section{Solution of the Schrödinger Equation}

Motion of the nonrelativistic spinless particles is described by the Schrödinger equation and in the existence of the external electromagnetic fields it is given by (we take $\hbar=1$ )

$$
\left[\frac{(\vec{P}-e \vec{A})^{2}}{2 m}\right] \Phi=\left(i \partial_{t}-e \phi\right) \Phi
$$

where $e$ is charge, $m$ is mass of the particle, $\phi$ is scalar potential, and $\vec{A}$ is the vector electromagnetic potential. In the following steps we solve the Schrödinger equation for different orientations of the external fields.

Case $(i)(\vec{E} \| \vec{B})$. We define the wave function by

$$
\Phi_{\|}=e^{i\left(x P_{x}-\varepsilon t\right)} C(y) D(z) .
$$

Then, (1) becomes

$$
[\widehat{U}(y)+\widehat{V}(z)] C(y) D(z)=0,
$$

where $\widehat{U}(y)=\widehat{P}_{y}^{2}+\left(P_{x}-\left(e B_{0} / \alpha\right) e^{-\alpha y}\right)^{2}$ and $\widehat{V}(z)=\widehat{P}_{z}^{2}-2 m(\varepsilon+$ $\left.e E_{0} e^{-\alpha z}\right)$. It can be separated into two independent equations given by

$$
\begin{aligned}
& {[\widehat{U}(y)-b] C(y)=0,} \\
& {[\widehat{V}(z)+b] D(z)=0,}
\end{aligned}
$$

where $b$ is a constant obtained from the separation.

By introducing the variable $\rho=2 e B_{0} e^{-\alpha y} / \alpha^{2}$ and writing $C(\rho)=\rho^{-1 / 2} f(\rho)$, (4) becomes

$$
\left\{\frac{d^{2}}{d \rho^{2}}+\frac{1}{\rho^{2}}\left(\frac{1}{4}-\frac{P_{x}^{2}-b}{\alpha^{2}}\right)+\frac{P_{x}}{\alpha} \frac{1}{\rho}-\frac{1}{4}\right\} f(\rho)=0,
$$

which is the Whittaker equation [12] whose solutions are the Whittaker functions represented by $W_{\kappa, \mu}(\rho)$. Therefore, exact solution for (4) is

$$
C(\rho)=\rho^{-1 / 2} W_{\kappa, \mu}(\rho)
$$

where $\kappa$ and $\mu$ are given by $\kappa=P_{x} / \alpha$ and $\mu= \pm \sqrt{P_{x}^{2}-b} / \alpha$.

For the requirement that the Whittaker functions must be bounded for the variable [12] we have

$$
\frac{1}{2}+\mu-\kappa=-n
$$

where $n$ is a positive integer. By using the explicit values of $\kappa$ and $\mu$ in that expression we obtain the below condition for $b$ constant

$$
b=\alpha N\left[2 P_{x}-\alpha N\right],
$$

which reveals that the motion of particle in $(x, y)$ plane is quantized. Here, the definition $N=(n+1 / 2)$ is made. The

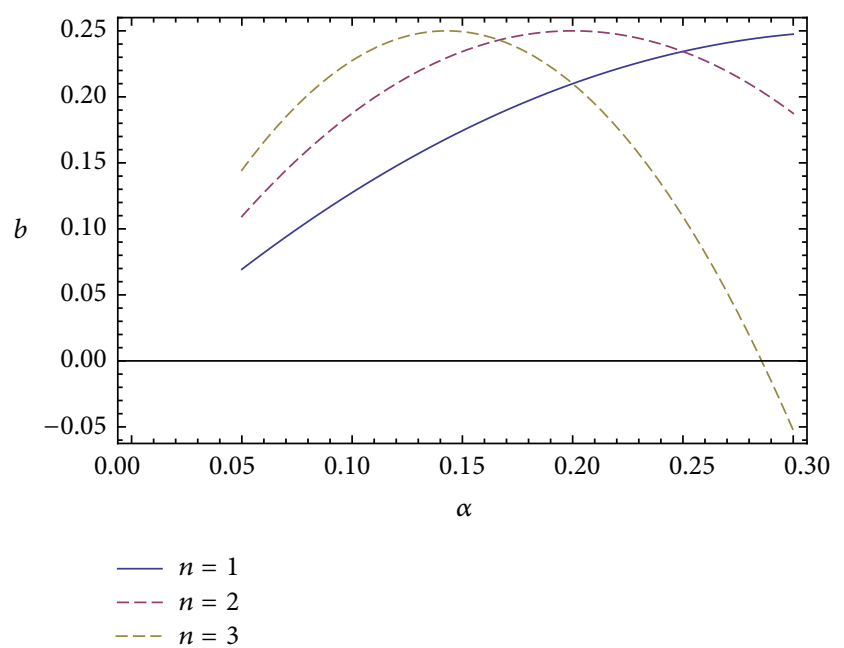

FIGURE 1: Variation of the $b$ constant as a function of the $\alpha$ parameter for the first three values of quantum number $n$. We set $m=1$ and $P_{x}=0.5$.

variation of the $b$ constant against $\alpha$ parameter is depicted in Figure 1.

Equation (5) can be solved in the same manner. After defining a new variable in the form $\beta=-2 m e E_{0} e^{-\alpha z}$, it turns into

$$
\left\{\beta^{2} \frac{d^{2}}{d \beta^{2}}+\beta \frac{d}{d \beta}+\frac{2 m \varepsilon-b}{\alpha^{2}}-\frac{\beta}{\alpha^{2}}\right\} D(\beta)=0 .
$$

The solution of this equation is [13]

$$
D(z)=Z_{\nu}\left( \pm \frac{2 i}{\alpha} \beta^{1 / 2}\right)
$$

where $Z_{\nu}$ are the Bessel functions and $\nu=( \pm(2 / \alpha) \sqrt{b-2 m \varepsilon})$.

Then, the exact solution is written in the form

$$
\Phi_{\|}=N_{1} e^{i\left(x P_{x}-\varepsilon t\right)} \rho^{-1 / 2} W_{\kappa, \mu}(\rho) Z_{\nu}\left( \pm \frac{2 i}{\alpha} \beta^{1 / 2}\right)
$$

where $N_{1}$ is the normalization constant. By setting $v$ to a constant value, say $k$, and using the expression obtained for $b$ we get the energy eigenvalues for the Schrödinger equation as follows:

$$
\varepsilon_{\| \text {Sch. }}=\frac{1}{2 m}\left\{\alpha N\left[2 P_{x}-\alpha N\right]-\frac{\alpha^{2} k^{2}}{4}\right\} .
$$

This variation of the energy spectrum for nonrelativistic spinless particles as the function of the $\alpha$ parameter is given in Figure 2.

Case (ii) $(\vec{E} \perp \vec{B})$. By defining the wave function as

$$
\Phi_{\perp}=e^{i\left(x P_{x}+z P_{z}-\varepsilon t\right)} F(y)
$$




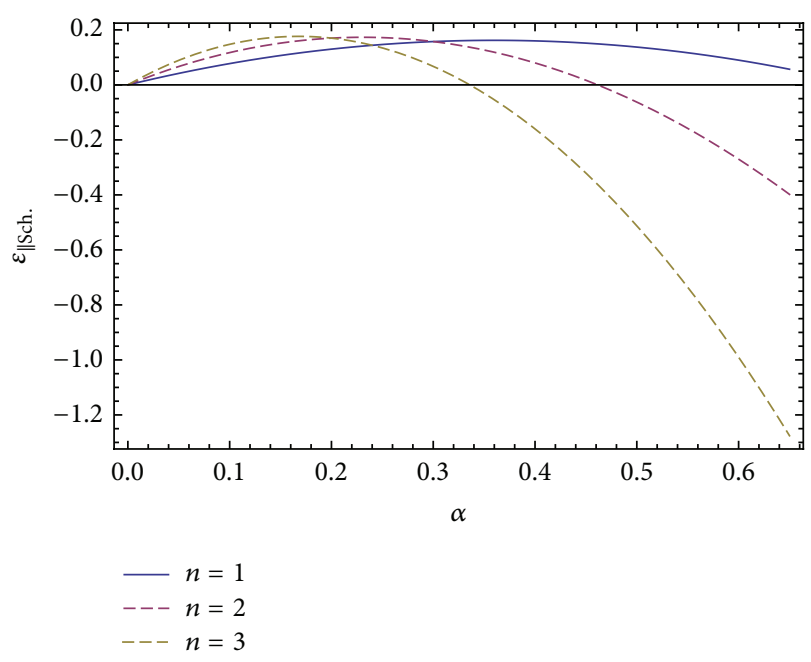

FIGURE 2: Dependence of the energy of nonrelativistic particles on the $\alpha$ parameter (in units of $m$ ) in the case of the parallel electromagnetic fields for the first three values of the quantum number $n$. We set $m=1, k=1$, and $P_{x}=0.6$.

the Schrödinger equation takes the following form:

$$
\begin{aligned}
& {\left[\frac{d^{2}}{d y^{2}}-\frac{e^{2} B_{0}^{2} e^{-2 \alpha y}}{\alpha^{2}}+\frac{2 e P_{x} B_{0} e^{-\alpha y}}{\alpha}-2 m e E_{0} e^{-\alpha y}\right.} \\
& \left.+\left(2 m \varepsilon-P_{z}^{2}-P_{x}^{2}\right)\right] F(y)=0 .
\end{aligned}
$$

Reusing $\rho=2 e B_{0} e^{-\alpha y} / \alpha^{2}$ and writing $F(\rho)=\rho^{-1 / 2} f(\rho)$, we obtain the solution of the above equation in terms of the Whittaker functions:

$$
f(\rho)=W_{\widetilde{\kappa}, \widetilde{\mu}}(\rho),
$$

where $\tilde{\kappa}=\left(P_{x} / \alpha-m E_{0} / B_{0}\right)$ and $\tilde{\mu}= \pm \sqrt{P_{x}^{2}+P_{z}^{2}-2 m \varepsilon} / \alpha$. by

Then, exact solution of the Schrödingr equation is given

$$
\Phi_{\perp}=N_{2} e^{i\left(x P_{x}+z P_{z}-\varepsilon t\right)} \rho^{-1 / 2} W_{\widetilde{\mathcal{K}}, \widetilde{\mu}}(\rho)
$$

where $N_{2}$ is the normalization constant. By using (8) for $\widetilde{\mathcal{\kappa}}$ and $\widetilde{\mu}$, we obtain the quantized kinetic energy spectrum as follows:

$$
\varepsilon_{\perp \text { Sch. }}=\frac{1}{2 m}\left\{P_{x}^{2}+P_{z}^{2}-\left[P_{x}-\alpha(\widetilde{N}+\lambda)\right]^{2}\right\}
$$

where $\lambda=m E_{0} / B_{0}, \widetilde{N}=(\widetilde{n}+1 / 2)$, and $\widetilde{n}$ is a positive integer. The change of the energy spectrum due to the $\alpha$ parameter for the orthogonal electromagnetic fields is presented in Figure 3.

The energy spectrum obtained above shows that the magnetic field exists from the beginning of the space. $B_{0}$ has to take nonvanishing values lest the kinetic energy becomes infinite. Also the energy of the particles reduces by the increasing $E_{0}$. However, there should be a limitation on the increasing of the strength of the electric field so that the

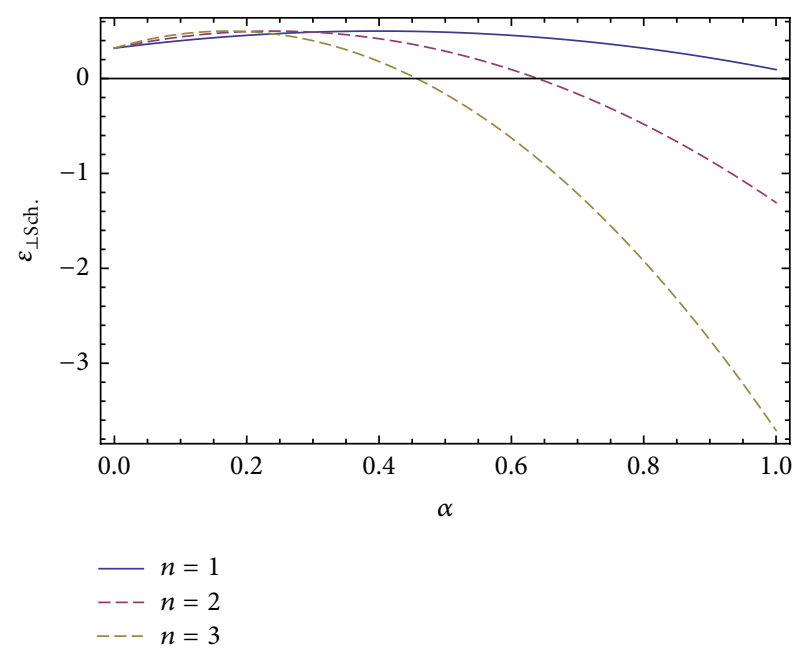

FIGURE 3: Dependence of the energy of the nonrelativistic particles on the $\alpha$ parameter (in units of $m$ ) in the case of the orthogonal electromagnetic fields for the first three values of the quantum number $n$. We set $m=1, P_{x}=0.6, P_{z}=0.8, E_{0}=0.5$, and $B_{0}=500$.

energy of particle has to be positive. Similar to the situation in classical motions electric field must be set to a much smaller value compared to the value of the magnetic field to ensure the kinetic energy is positive. This requirement can be seen explicitly by writing (18) in the limit of $E_{0} \gg B_{0}$,

$$
\varepsilon_{\perp \text { Sch. }} \simeq \frac{1}{2 m}\left\{P_{z}^{2}+2 \alpha \widetilde{N} P_{x}-\alpha^{2}\left[\widetilde{N}^{2}+\lambda^{2}\right]\right\}
$$

It is clear from the resulting expression that the Schrödinger equation cannot be applied in this limit since the energy becomes comparable to the rest mass energy of the particle. For the nonrelativistic case we know that the difference between the total energy and rest mass energy is very small which means that the kinetic energy has to be very small compared to the rest mass energy.

\section{Solution of the Klein-Gordon Equation}

The Klein-Gordon equation for the relativistic spin-0 particles like pions or kaons interacting with electromagnetic fields is given by [14]

$$
\left[(i \vec{\nabla}+e \vec{A})^{2}+m^{2}\right] \psi=\left(i \partial_{t}-e A_{0}\right)^{2} \psi
$$

where $e$ and $m$ are charge and mass of the particle and $A_{\mu}=$ $\left(A_{0}, \vec{A}\right)$ is the four-vector electromagnetic potential. We use the Heaviside units, $\hbar=c=1$.

Case (i) $(\vec{E} \| \vec{B})$. For that case we take the wave function in the form

$$
\psi_{\|}=e^{i\left(x P_{x}-\varepsilon t\right)} G(y) H(z),
$$

and (20) turns into

$$
\left[\widehat{I}(y)+\widehat{J}(z)-m^{2}\right] G(y) H(z)=0,
$$




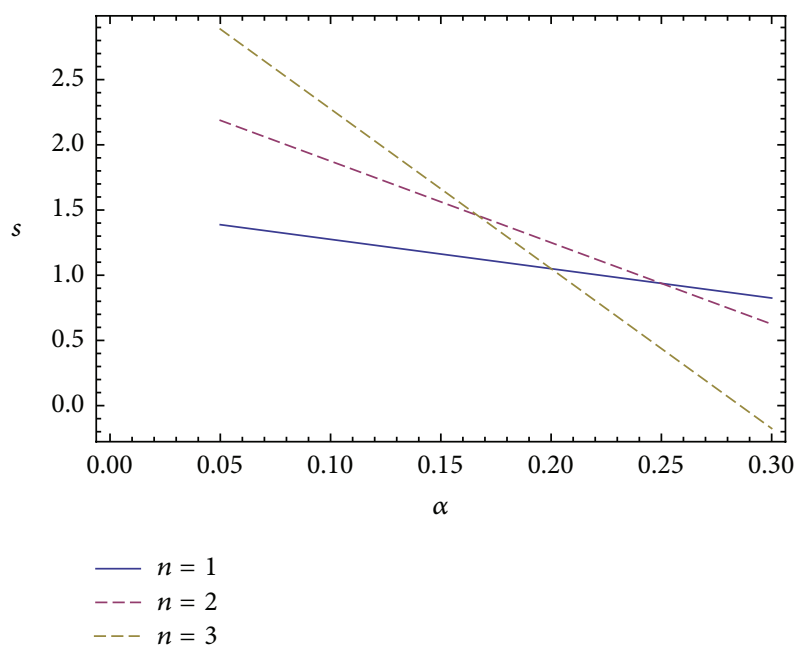

FIGURE 4: Variation of the $s$ constant as a function of the $\alpha$ parameter for the first three values of quantum number $n$. We set $P_{x}=0.5$.

where $\widehat{I}(y)=d^{2} / d y^{2}-\left(P_{x}-\left(e B_{0} / \alpha\right) e^{-\alpha y}\right)^{2}$ and $\widehat{J}(z)=d^{2} /$ $d z^{2}+\left(\varepsilon+e E_{0} e^{-\alpha z}\right)^{2}$.

This gives two independent equations in the forms

$$
\begin{gathered}
{[\widehat{I}(y)+s] G(y)=0,} \\
{\left[\widehat{J}(z)-\left(s+m^{2}\right)\right] H(z)=0,}
\end{gathered}
$$

where $s$ is the separation constant for the Klein-Gordon equation.

One more time using the variable $\rho$, and writing $G(\rho)=$ $\rho^{-1 / 2} g(\rho)$, (23) reduces to the Whittaker equation and the solution is given by

$$
G(\rho)=\rho^{-1 / 2} W_{\kappa, \tau}(\rho),
$$

where $\kappa=P_{x} / \alpha$ and $\tau= \pm \sqrt{P_{x}^{2}-s} / \alpha$.

By using the condition given in (8) we obtain

$$
s=N \alpha\left[2 P_{x}-\alpha N\right] .
$$

The variation of the $s$ constant against $\alpha$ parameter is seen in Figure 4.

In order to solve (24) we define $\widetilde{\rho}=\left(2 e i E_{0} / \alpha\right) e^{-\alpha z}$ and write $H(\widetilde{\rho})=\widetilde{\rho}^{-1 / 2} h(\widetilde{\rho})$. With these definitions the resulting equation becomes the Whittaker equation and the solution is

$$
h(\widetilde{\rho})=W_{\sigma, \eta}(\widetilde{\rho}),
$$

where $\sigma=i \varepsilon / \alpha$ and $\eta= \pm \sqrt{\left(s+m^{2}\right)-\varepsilon^{2}} / \alpha$.

Thus, the exact solution of the Kein-Gordon equation for parallel electromagnetic fields is given by

$$
\psi_{\|}=N_{3} e^{i\left(x P_{x}-\varepsilon t\right)} \rho^{-1 / 2} \widetilde{\rho}^{-1 / 2} W_{\sigma, \eta}(\widetilde{\rho}) W_{\kappa, \tau}(\rho),
$$

where $N_{3}$ is the normalization constant.

Setting the $\eta$ to a constant value, say $w$, and using the expression obtained in (26) for $s$, we get the following energy

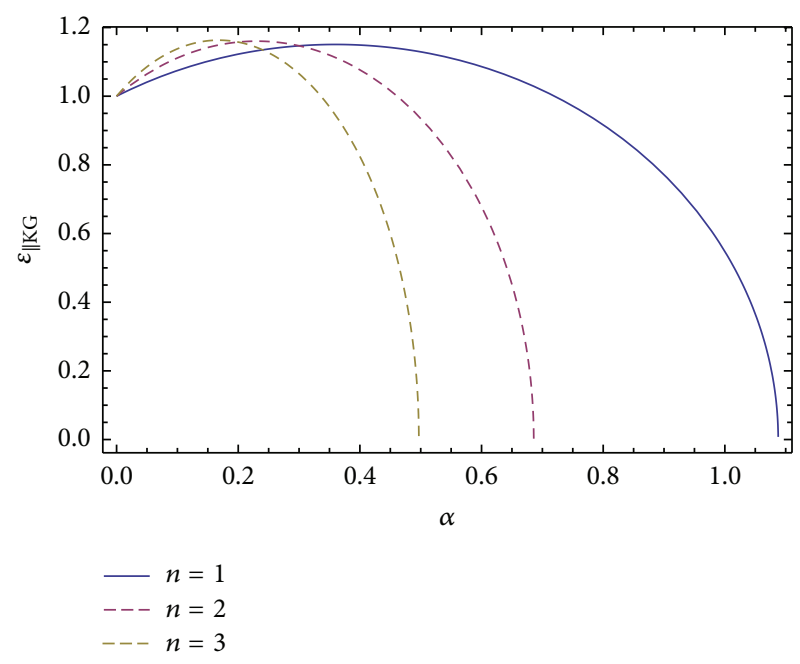

FIGURE 5: Dependence of the energy of the relativistic spinless particles on the $\alpha$ parameter (in units of $m$ ) in the case of the orthogonal external fields for the first three values of the quantum number $n$. We set $m=1, \omega=0.5$, and $P_{x}=0.6$.

TABLE 1: Comparison of the energy spectrum of the spinless particles for parallel electromagnetic fields as a function of the $\alpha$ parameter for the nonrelativistic and the relativistic cases. We set $m=1$ and $P_{x}=0.6$.

\begin{tabular}{cccc}
\hline$n$ & $\alpha$ & $\varepsilon_{\|_{\text {Sch. }}}$ & $\mathcal{E}_{\|_{\text {KG }}}$ \\
\hline \multirow{3}{*}{1} & 0.1 & 0.0775 & 1.07121 \\
& 0.2 & 0.1300 & 1.10905 \\
& 0.3 & 0.1575 & 1.11692 \\
& 0.4 & 0.1600 & 1.09545 \\
& 0.5 & 0.1375 & 1.4283 \\
\hline \multirow{3}{*}{2} & 0.1 & 0.1175 & 1.10793 \\
& 0.2 & 0.1700 & 1.14455 \\
& 0.3 & 0.1575 & 1.11692 \\
& 0.4 & 0.0800 & 1.01980 \\
& 0.5 & -0.0625 & 0.829156 \\
\hline \multirow{3}{*}{3} & 0.1 & 0.1475 & 1.13468 \\
& 0.2 & 0.1700 & 1.14455 \\
& 0.3 & 0.0675 & 1.03320 \\
& 0.4 & -0.1600 & 0.748331 \\
\hline
\end{tabular}

spectrum for the Klein-Gordon equation in the case of parallel electromagnetic fields:

$$
\varepsilon_{\|_{\mathrm{KG}}}= \pm \sqrt{2 N \alpha P_{x}-\alpha^{2}\left(N^{2}+w^{2}\right)+m^{2}} .
$$

The energy eigenvalues depending on the $\alpha$ parameter for the parallel electromagnetic fields are seen in Figure 5.

Some numerical results for the energy eigenvalues of the spinless particles subjected to the parallel electric and magnetic fields are listed in Table 1 . We notice that the energy eigenvalues for the Schrödinger and Klein-Gordon particles present opposite behavior depending on the variation of $\alpha$ 
parameter. This means that as the external fields become weak due to the increasing of the $\alpha$, the energy of the nonrelativistic particles increases whereas the energy of the relativistic particles decreases. Nevertheless this behavior is not stable due to the increasing $n$ values.

Case (ii) $(\vec{E} \perp \vec{B})$. By defining the wave function as

$$
\psi_{\perp}=e^{i\left(x P_{x}+z P_{z}-\varepsilon t\right)} M(y)
$$

the Klein-Gordon equation takes the following form:

$$
\widehat{T}(y) M(y)=0,
$$

where

$$
\begin{aligned}
\widehat{T}(y)= & \widehat{P}_{y}^{2}+\frac{e^{2}}{\alpha^{2}}\left(B_{0}^{2}-\alpha^{2} E_{0}^{2}\right) e^{-2 \alpha y}-\frac{2 e}{\alpha}\left(P_{x} B_{0}-\varepsilon E_{0} \alpha\right) e^{-\alpha y} \\
& +P_{x}^{2}+P_{z}^{2}+m^{2}-\varepsilon^{2} .
\end{aligned}
$$

We analyse this equation by considering the relation of strengths of electric and magnetic fields.

For the first situation let their magnitude be equal. In addition to that, $\widehat{T}(y)$ becomes independent of $y$ in case of the $\varepsilon=P_{x}$ and motion of particle becomes unrestricted. In order for the situation to be physical, the total energy $\varepsilon$ must be greater than the momentum of the particle unless its mass equals zero. By this requirement, $\widehat{T}(y)$ has to depend on the $y$. Therefore the form of the $\widehat{T}(y)$ should be as follows:

$$
\widehat{T}(y)=\widehat{P}_{y}^{2}-\frac{2 e}{\alpha}\left(P_{x} B_{0}-\varepsilon E_{0} \alpha\right) e^{-\alpha y}+P_{x}^{2}+P_{z}^{2}+m^{2}-\varepsilon^{2} .
$$

In that case by defining a new variable as $\xi=e^{-\alpha y}$, (31) takes the form

$$
\begin{gathered}
\left\{\xi^{2} \frac{d^{2}}{d \xi^{2}}+\xi \frac{d}{d \xi}+\frac{2 e\left(P_{x} B_{0}-\varepsilon E_{0} \alpha\right)}{\alpha^{3}} \xi\right. \\
\left.+\frac{\varepsilon^{2}-m^{2}-P_{x}^{2}-P_{z}^{2}}{\alpha^{2}}\right\} M(\xi)=0
\end{gathered}
$$

whose solution is given in terms of the Bessel functions as follows:

$$
M(\xi)=Z_{\widetilde{\nu}}\left(\sqrt{\frac{8 e\left(P_{x} B_{0}-\varepsilon E_{0} \alpha\right)}{\alpha^{3}}} \xi^{1 / 2}\right),
$$

where $\widetilde{\nu}=\mp 2 \sqrt{m^{2}+P_{x}^{2}+P_{z}^{2}-\varepsilon^{2}} / \alpha$.

Then, the exact solution is given by

$$
\psi_{\perp}=e^{i\left(x P_{x}+z P_{z}-\varepsilon t\right)} Z_{\widetilde{v}}\left(\sqrt{\frac{8 e\left(P_{x} B_{0}-\varepsilon E_{0} \alpha\right)}{\alpha^{3}}} \xi^{1 / 2}\right) .
$$

For the other situation we consider the fields having different magnitudes. By keeping the form of (32) and

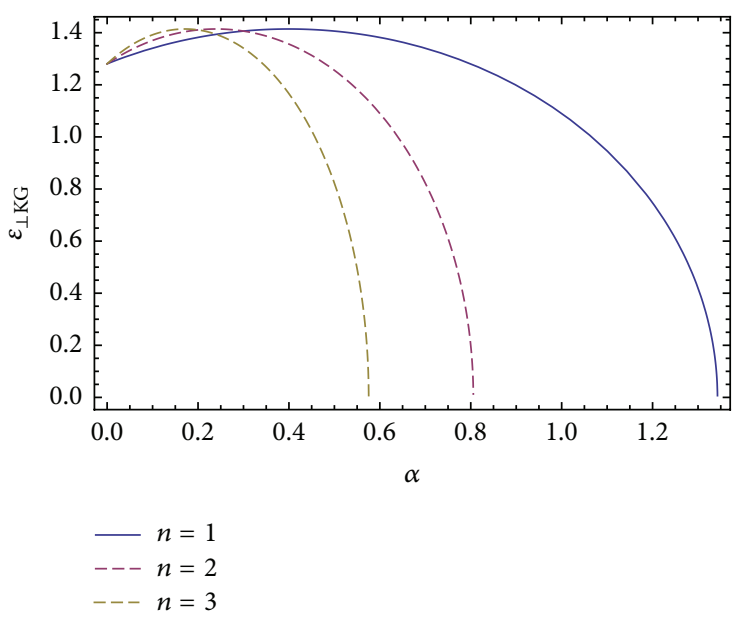

Figure 6: Dependence of the energy of the relativistic spinless particles on the $\alpha$ parameter (in units of $m$ ) in the case of the orthogonal external fields for the first three values of the quantum number $n$. We set $m=1, P_{x}=0.6, P_{z}=0.8, E_{0}=0.5$, and $B_{0}=500$.

defining a new variable as $\tilde{\xi}=2 e \sqrt{B_{0}^{2}-\alpha^{2} E_{0}^{2}} e^{-\alpha y} / \alpha^{2}$, and writing $M(\tilde{\xi})=\widetilde{\xi}^{-1 / 2} \Lambda(\widetilde{\xi})$, the solution is found in terms of the Whittaker functions and given by

$$
M(\widetilde{\xi})=\widetilde{\xi}^{-1 / 2} W_{\phi, \pi}(\widetilde{\xi})
$$

where $\phi=\left(P_{x} B_{0}-\alpha \varepsilon E_{0}\right) / \alpha \sqrt{B_{0}^{2}-\alpha^{2} E_{0}^{2}}$ and $\pi=$ $\pm \sqrt{m^{2}+P_{x}^{2}+P_{z}^{2}-\varepsilon^{2}} / \alpha$.

Therefore, exact solution for the Klein-Gordon equation is given as follows:

$$
\psi_{\perp}=N_{4} e^{i\left(x P_{x}+z P_{z}-\varepsilon t\right)} \widetilde{\xi}^{-1 / 2} W_{\phi, \pi}(\widetilde{\xi})
$$

where $N_{4}$ is the normalization constant. Using the condition given by (8) for $\phi$ and $\pi$, the quantized energy spectrum can be derived as follows:

$$
\begin{aligned}
\varepsilon_{\perp_{\mathrm{KG}}}= & \left(\alpha E_{0}\left(P_{x} B_{0}-\widetilde{N} \alpha \gamma\right)\right. \\
& \left.\quad \pm \gamma \sqrt{B_{0}^{2}\left(m^{2}+P_{x}^{2}+P_{z}^{2}\right)-\left(\widetilde{N} \alpha \gamma-P_{x} B_{0}\right)^{2}}\right) \\
& \times\left(B_{0}^{2}\right)^{-1},
\end{aligned}
$$

where $\widetilde{N}=(\widetilde{n}+1 / 2), \widetilde{n}$ is an integer, and $\gamma=\sqrt{B_{0}^{2}-\alpha^{2} E_{0}^{2}}$. Figure 6 shows the dependence of the energy spectrum of the relativistic spinless particles on the $\alpha$ parameter. Energy eigenvalues decrease with the increasing $\alpha$ values that weaken the electromagnetic field strength.

Some numerical results for the energy eigenvalues of the particles in the case of the orthogonal electromagnetic fields are presented in Table 2 . The table reveals that as the external fields become weak, both the nonrelativistic and the relativistic spinless particles become more energetic up to 
TABLE 2: Comparison of the energy spectrum of the spinless particles for orthogonal electromagnetic fields as a function of the $\alpha$ parameter for the nonrelativistic and the relativistic cases. We set $m=1, P_{x}=0.6, P_{z}=0.8, E_{0}=0.5$, and $B_{0}=500$.

\begin{tabular}{cccc}
\hline$n$ & $\alpha$ & $\varepsilon_{\perp_{\text {Sch. }}}$ & $\varepsilon_{\perp_{\mathrm{KG}}}$ \\
\hline \multirow{3}{*}{1} & 0.1 & 0.398795 & 1.34075 \\
& 0.2 & 0.45506 & 1.38209 \\
& 0.3 & 0.488795 & 1.40628 \\
& 0.4 & 0.5 & 1.41421 \\
& 0.5 & 0.488675 & 1.40616 \\
\hline & 0.1 & 0.438785 & 1.37025 \\
& 0.2 & 0.49502 & 1.41069 \\
& 0.3 & 0.488705 & 1.40619 \\
& 0.4 & 0.41984 & 1.35631 \\
& 0.5 & 0.288425 & 1.25566 \\
\hline \multirow{3}{*}{3} & 0.1 & 0.468775 & 1.39197 \\
& 0.2 & 0.49498 & 1.41065 \\
& 0.3 & 0.398615 & 1.34057 \\
& 0.4 & 0.17968 & 1.16587 \\
& 0.5 & -0.161825 & 0.822529 \\
\hline
\end{tabular}

some certain values of the $\alpha$ and later their energy starts to decrease. This character is also applicable for $n=2$ and $n=3$.

The total energy of the relativistic spinless particles reduces to the following form for pure magnetic fields:

$$
\varepsilon_{\perp_{\mathrm{KG}}}= \pm \sqrt{\left(m^{2}+P_{z}^{2}\right)+2 \alpha P_{x} \widetilde{N}-\alpha^{2} \widetilde{N}^{2}} .
$$

\section{Conclusion}

We analysed the motion of the spinless particles in parallel and orthogonal electric and magnetic fields. Investigation was performed for nonrelativistic and relativistic cases to evaluate the contributions coming from the relativistic effects. It is found that, in the case of $\vec{E} \| \vec{B}$, the relativistic effects arise only for the motion in the $z$-direction. In that case the Whittaker functions that occurred in the relativistic solutions are replaced by the Bessel functions for nonrelativistic solutions. In case of the orthogonal fields $\vec{E} \perp \vec{B}$, exact solutions of the Schrödinger and Klein-Gordon equations are found in terms of the Whittaker functions for unequal strength of the fields. For the relativistic case due to the equal strength of the fields the solutions are obtained in terms of the Bessel functions. In the case of the parallel fields the motion has a more complex form and has been described by the product of two functions. On account of restricted knowledge of recurrence relation between the product of the Whittaker and Bessel functions or between the unequal $\mu$ indexed Whittaker functions, we left the exact solutions in the obtained forms. This is not the case for the orthogonal fields in which the quantized energy spectrum was obtained directly by using the condition given with (8) for both the Schrödinger and Klein-Gordon equations.

The energy eigenvalues against the $\alpha$ parameter were drawn for nonrelativistic and relativistic cases in Figures 2,
3,5 , and 6 . These figures show us that the number of the bound states for Schrödinger and Klein-Gordon equations in the presence of the exponentially decreasing electromagnetic fields is limited due to the increasing values of the $\alpha$ that weaken the fields' strength. The energy eigenvalues decrease by increasing values of the $\alpha$ and become negative finally. At these values of the $\alpha$ the particles are not bounded anymore. By considering the energy expressions obtained for the Schrödinger and the Klein-Gordon equations for each case of the orientation of the fields, the $\alpha$ values that cause the energy to be zero can be derived easily. These values are also seen from Figures 2, 3, 5, and 6. The important point here is that although the values of the $\alpha$ that set the energy to zero in the case of the parallel electromagnetic fields are independent of the $m, E_{0}$, and $B_{0}$ for the spinless particles, they depend on these parameters in the case of the orthogonal electromagnetic fields.

In order to see the correlation between the energy spectrum of both cases, we may write their limiting expressions as follows:

$$
\left(\varepsilon_{\| \mathrm{KG}}-m\right) \approx \varepsilon_{\| S \mathrm{Sch} .}-\frac{1}{8 m^{3}}\left\{\alpha N\left[2 P_{x}-\alpha N\right]-\frac{\alpha^{2} k^{2}}{4}\right\},
$$

where the second term of the right side corresponds to the contribution of the relativity, and

$$
\begin{aligned}
& \left(\varepsilon_{\perp \mathrm{KG}}-m\right) \\
& \approx \varepsilon_{\perp \text { Sch. }}-\frac{1}{8 m^{3}}\left(P_{z}^{2}+2 \alpha \widetilde{N} P_{x}-\alpha^{2} \widetilde{N}^{2}\right)^{2} \\
& +\frac{1}{2 m}\left(\frac{\alpha E_{0}}{B_{0}}\right)^{2} \\
& \times\left\{-\alpha \widetilde{N} P_{x}+\alpha^{2} \widetilde{N}^{2}\right. \\
& +\frac{1}{4 m^{2}}\left(P_{z}^{2}+2 \alpha \widetilde{N} P_{x}-\alpha^{2} \widetilde{N}^{2}\right) \\
& \left.\times\left[-\alpha \widetilde{N} P_{x}+\alpha^{2} \widetilde{N}^{2}-\frac{1}{2}\left(P_{z}^{2}+2 \alpha \widetilde{N} P_{x}-\alpha^{2} \widetilde{N}^{2}\right)\right]\right\},
\end{aligned}
$$

for which we impose the below two conditions:

(1) $\left(\alpha E_{0} / B_{0}\right)^{2}<1$,

(2) $\left(1 / m^{2}\right)\left[\left(P_{z}^{2}+2 \alpha \widetilde{N} P_{x}-\alpha^{2} \widetilde{N}^{2}\right)+\left(\alpha E_{0} / B_{0}\right)^{2}\left(-\alpha \widetilde{N} P_{x}+\right.\right.$ $\left.\left.\alpha^{2} \widetilde{N}^{2}\right)\right]<1$. The second term of the right side of (42) is the contribution coming from the relativity and the third term is the contribution of the external electromagnetic fields.

As a final remark by considering (9) and (26) we can say that the motion of the spinless particles in the case of parallel fields is quantized in $(x, y)$ plane for the nonrelativistic and the relativistic cases. 


\section{Conflict of Interests}

The authors declare that there is no conflict of interests regarding the publication of this paper.

\section{References}

[1] P. J. Redmond, "Solution of the Klein-Gordon and Dirac equations for a particle with a plane electromagnetic wave and a parallel magnetic field," Journal of Mathematical Physics, vol. 6, pp. 1163-1169, 1965.

[2] R. L. Liboff, "Brownian motion of charged particles in crossed electric and magnetic fields," Physical Review, vol. 141, no. 1, pp. 222-227, 1966.

[3] F. Occhionero and M. Demianski, "Electric fields in rotating, magnetic," Physical Review Letters, vol. 23, pp. 1128-1130, 1969.

[4] L. Lam, "Motion in electric and magnetic fields. I. KleinGordon particles," Journal of Mathematical Physics, vol. 12, no. 2, pp. 299-303, 1971.

[5] M. Grewing and H. Heintzmann, "Charged particle motion in superstrong electromagnetic fields," Physics Letters A, vol. 42, no. 4, pp. 325-326, 1972.

[6] J. Bergou and F. Ehlotzky, "Relativistic quantum states of a particle in an electromagnetic plane wave and a homogeneous magnetic field," Physical Review A, vol. 27, no. 5, pp. 2291-2296, 1983.

[7] G. Ivanovski, D. Jakimovski, and V. Sopova, "Energy levels of a charged particle in a homogeneous electric field orthogonal to a piecewise homogeneous magnetic field," Physics Letters A, vol. 183, no. 1, pp. 24-28, 1993.

[8] V. M. Villalba and R. Pino, "Energy spectrum of a relativistic two-dimensional hydrogen-like atom in a constant magnetic field of arbitrary strength," Physica E: Low-Dimensional Systems and Nanostructures, vol. 10, no. 4, pp. 561-568, 2001.

[9] C.-M. Chiang and C.-L. Ho, "Charged particles in external fields as physical examples of quasi-exactly-solvable models: a unified treatment," Physical Review A, vol. 63, no. 6, Article ID 062105, 2001.

[10] A. Rutkowski and A. Poszwa, "Relativistic corrections for a twodimensional hydrogen-like atom in the presence of a constant magnetic field," Physica Scripta, vol. 79, no. 6, Article ID 065010, 2009.

[11] R. Burman, "Exponentially varying electromagnetic fields in a stratified magnetoplasma," Proceedings of the IEEE, vol. 54, no. 6, pp. 888-889, 1966.

[12] M. Abramowitz and I. A. Stegun, Handbook of Mathematical Functions, Dover, New York, NY, USA, 1970.

[13] E. Komke, Mathematik und Ihre Anwedungen in Physik und Technik, Geest und Portig, Leipzig, Germany, 1967.

[14] W. Greiner, Relativistic Quantum Mechanics, Springer, Berlin, Germany, 1997. 

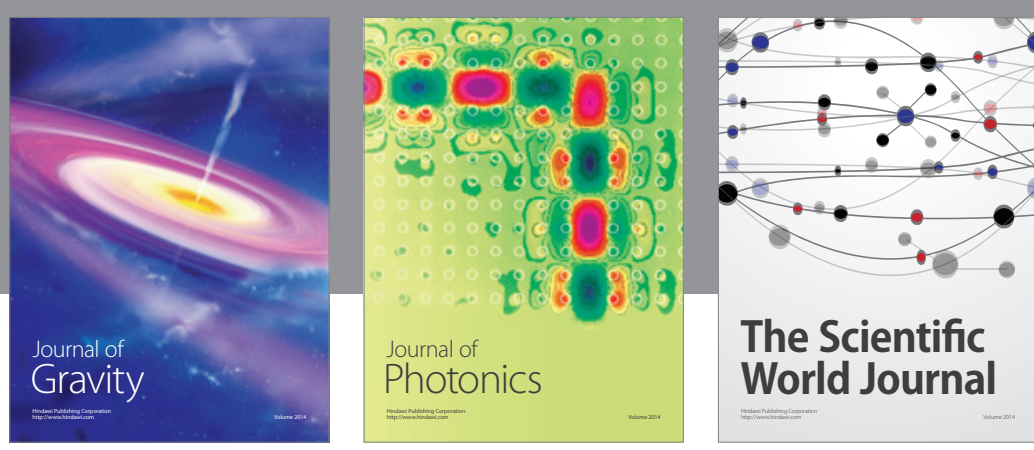

The Scientific World Journal
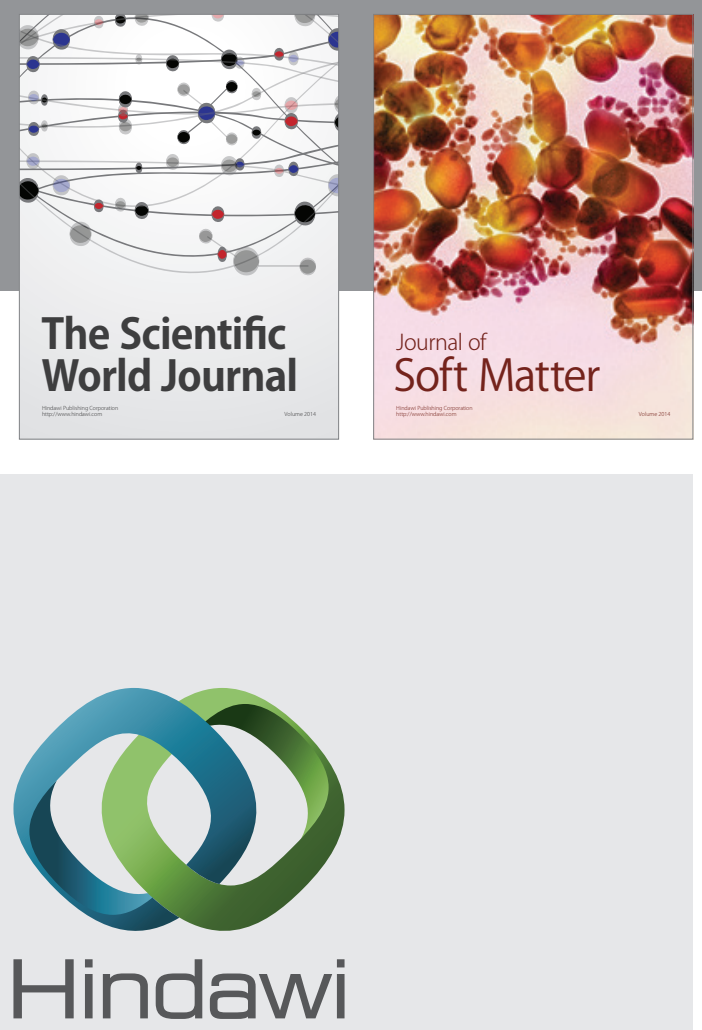

Submit your manuscripts at

http://www.hindawi.com

nternational Journal of

Statistical Mechanics
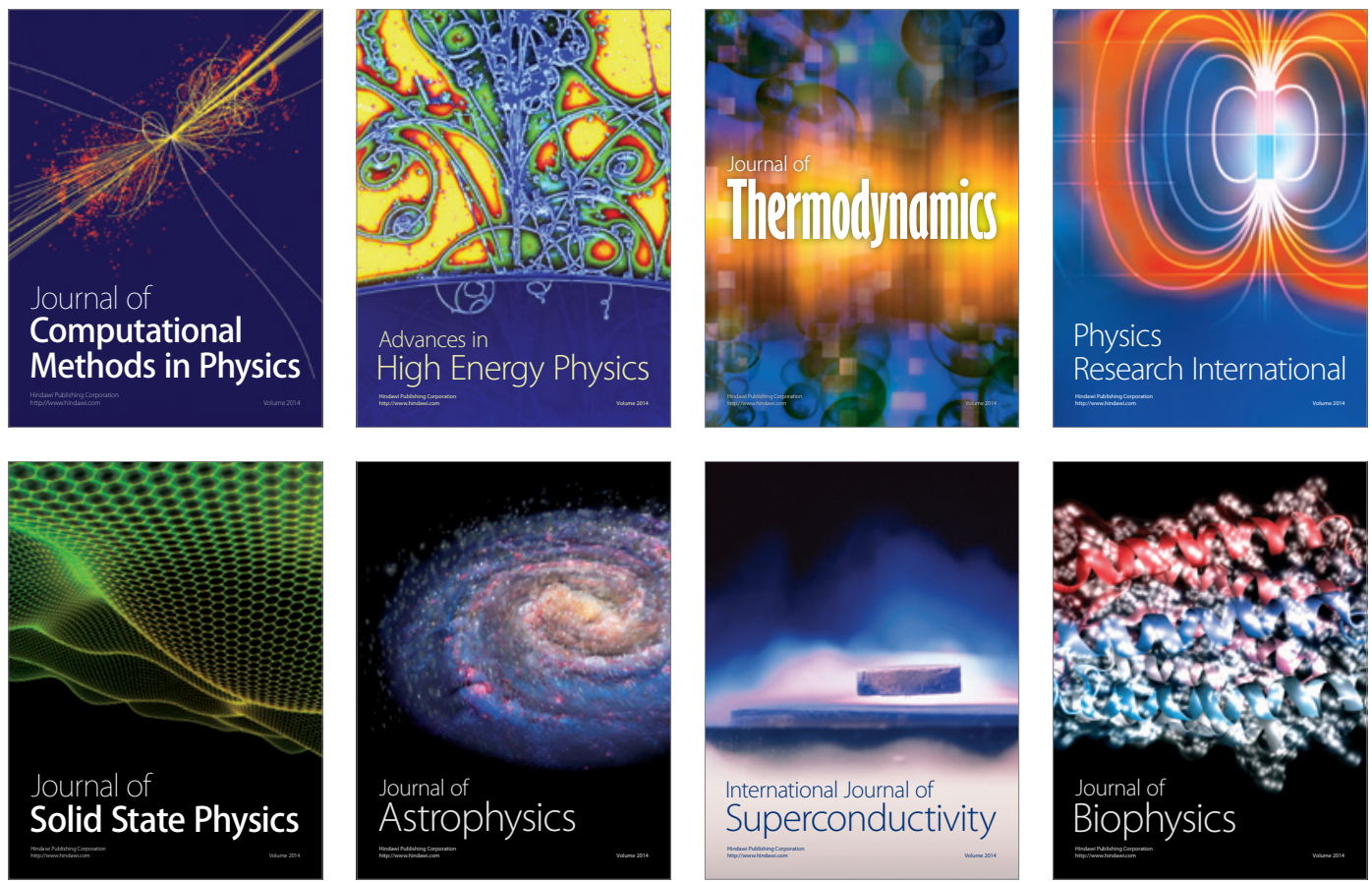
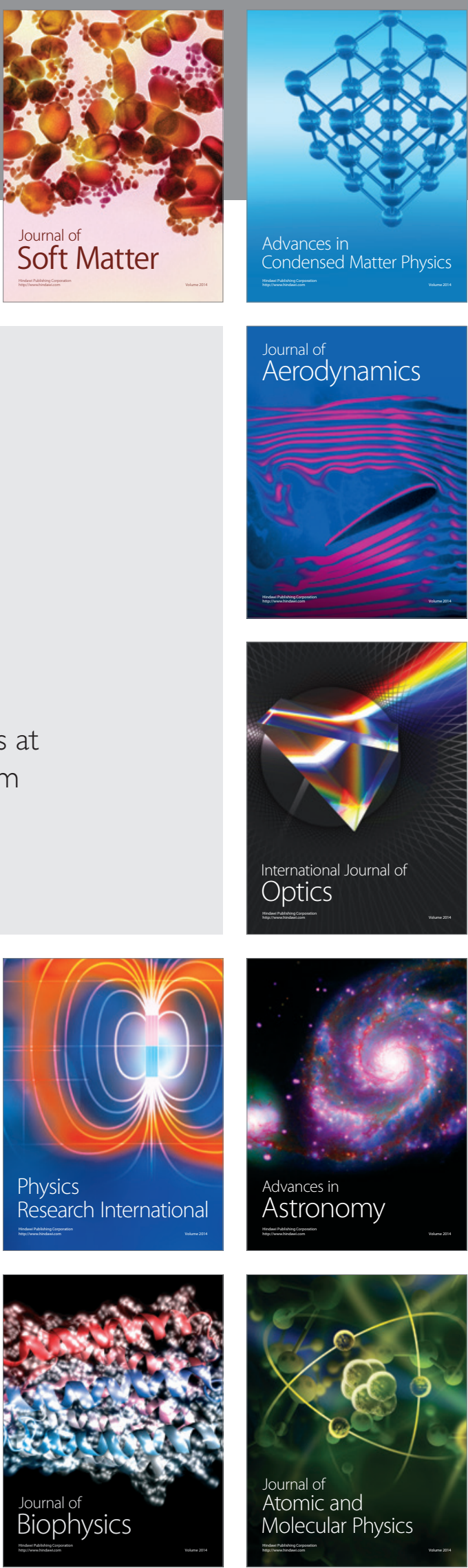